\title{
Visual distinguishability of polygons
}

\author{
ÁRPÁD KURUSA
}

\begin{abstract}
Considering equioptic curves of segments in the plane we prove that two polygons are distinguishable by their visual angles given on the boundary of an open domain that contains both polygons if the boundary either does not contain some exceptional types of arcs or is differentiable by order seven.
\end{abstract}

\section{Introduction}

The shadow picture [4] of a compact convex set $\mathcal{B}$ is defined at each point $P \in \mathbb{R}^{2} \backslash \mathcal{B}$ as the angle measure $\nu_{\mathcal{B}}(P)$ of the so called visual angle that $\mathcal{B}$ subtends at $P$. The point $P$ and the set $\mathcal{B}$ are usually called the source and the object of shadow picture $\nu_{\mathcal{B}}(P)$. The shadow picture is often called visual angle (sometimes this makes some confusion) or point projection [2]. The function $\nu_{\mathcal{B}}$ is usually called visual angle function.

The central problem in this subject is to show a set $\mathcal{S}$ of sources and a set $\mathcal{O}$ of objects that $\left.\mathcal{O} \ni \mathcal{B} \mapsto \nu_{\mathcal{B}}\right|_{\mathcal{S}}$ is injective. There are a number of such results in the literature; see [3], [4], [5], [6], [7] etc.

In this article we consider the distinguishability of convex polygons. Investigating the equioptics of pairs of segments allows us to drop the conditions of convexity and analyticity from earlier results given in [4].

\section{Equioptics of segments}

The equioptic of two compact convex sets is the set of those points from which their shadow pictures are equal. ${ }^{(2: 1)}$ The compoptic of two compact convex sets is

AMS Subject Classification (2000): 0052, 0054.

Key words and phrases: visual angle, shadow picture

$(2: 1)$ As the shadow picture of a segment is not well defined at the endpoints of the segment, we think of it as equal to any angle in $[0, \pi]$.

Beitr. Algebra Geom. 54 (2013), 659-667.

(c) Á. Kurusa 
the set of those points from which the sum of their shadow pictures is $\pi$ (these shadow pictures are said to be supplementary).

Lemma 2.1. The equioptic of two different segments is a union of subarcs of two cubic algebraic curves. The remaining subarcs of these two cubic algebraic curves constitute the compoptic of those segments.

Proof. Denote the different nondegenerate segments by $\overline{A B}$ and $\overline{C D}$. Let $\mathbf{m}$ be a unit normal vector of the plane. If the segment $\overline{A B}$ subtends the same shadow picture at $X$ as $\overline{C D}$ does, then

$$
(\overrightarrow{X A} \times \overrightarrow{X B})\langle\overrightarrow{X C}, \overrightarrow{X D}\rangle=\varepsilon(X)(\overrightarrow{X C} \times \overrightarrow{X D})\langle\overrightarrow{X A}, \overrightarrow{X B}\rangle,
$$

where

$$
\varepsilon(X)= \begin{cases}+1, & \text { if } \overrightarrow{X A} \times \overrightarrow{X B} \neq \mathbf{0} \text { and } \operatorname{sign}\langle\mathbf{m}, \overrightarrow{X A} \times \overrightarrow{X B}\rangle=\operatorname{sign}\langle\mathbf{m}, \overrightarrow{X C} \times \overrightarrow{X D}\rangle \\ +1, & \text { if } \overrightarrow{X A} \times \overrightarrow{X B}=\mathbf{0}, B \neq X \neq A \text { and } \operatorname{sign}\langle\overrightarrow{X A}, \overrightarrow{X B}\rangle=\operatorname{sign}\langle\overrightarrow{X C}, \overrightarrow{X D}\rangle, \\ -1, & \text { otherwise. }\end{cases}
$$

Obviously, $\varepsilon(X)$ is constant \pm 1 in every quadrant of the straight lines $A B$ and $C D$, and it changes its sign if and only if $X$ moves over one of the straight lines $A B$ and $C D$.

Fix an origin $O$ and let $\mathbf{x}=\overrightarrow{O X}, \mathbf{a}=\overrightarrow{O A}, \mathbf{b}=\overrightarrow{O B}, \mathbf{c}=\overrightarrow{O C}$ and $\mathbf{d}=\overrightarrow{O D}$. Then

$$
\begin{aligned}
& \overrightarrow{X A} \times \overrightarrow{X B}=(\mathbf{a}-\mathbf{x}) \times(\mathbf{b}-\mathbf{x})=\mathbf{x} \times(\mathbf{a}-\mathbf{b})+\mathbf{a} \times \mathbf{b}, \\
& \langle\overrightarrow{X A}, \overrightarrow{X B}\rangle=\langle\mathbf{a}-\mathbf{x}, \mathbf{b}-\mathbf{x}\rangle=|\mathbf{x}|^{2}-\langle\mathbf{x}, \mathbf{a}+\mathbf{b}\rangle+\langle\mathbf{a}, \mathbf{b}\rangle,
\end{aligned}
$$

and

$$
\begin{aligned}
& \overrightarrow{X C} \times \overrightarrow{X D}=(\mathbf{c}-\mathbf{x}) \times(\mathbf{d}-\mathbf{x})=\mathbf{x} \times(\mathbf{c}-\mathbf{d})+\mathbf{c} \times \mathbf{d} \\
& \langle\overrightarrow{X C}, \overrightarrow{X D}\rangle=\langle\mathbf{c}-\mathbf{x}, \mathbf{d}-\mathbf{x}\rangle=|\mathbf{x}|^{2}-\langle\mathbf{x}, \mathbf{c}+\mathbf{d}\rangle+\langle\mathbf{c}, \mathbf{d}\rangle
\end{aligned}
$$

therefore

$$
\begin{aligned}
|\mathbf{x}|^{2}\langle\mathbf{x}, & (\mathbf{a}-\mathbf{b}) \times \mathbf{m}\rangle+\langle\mathbf{a} \times \mathbf{b}, \mathbf{m}\rangle|\mathbf{x}|^{2}-\langle\mathbf{x},(\mathbf{a}-\mathbf{b}) \times \mathbf{m}\rangle\langle\mathbf{x}, \mathbf{c}+\mathbf{d}\rangle+ \\
& +\langle\mathbf{x},\langle\mathbf{c}, \mathbf{d}\rangle((\mathbf{a}-\mathbf{b}) \times \mathbf{m})-\langle\mathbf{a} \times \mathbf{b}, \mathbf{m}\rangle(\mathbf{c}+\mathbf{d})\rangle+\langle\mathbf{a} \times \mathbf{b}, \mathbf{m}\rangle\langle\mathbf{c}, \mathbf{d}\rangle \\
=\bar{\varepsilon}(\mathbf{x}) & \left(|\mathbf{x}|^{2}\langle\mathbf{x},(\mathbf{c}-\mathbf{d}) \times \mathbf{m}\rangle+\langle\mathbf{c} \times \mathbf{d}, \mathbf{m}\rangle|\mathbf{x}|^{2}-\langle\mathbf{x},(\mathbf{c}-\mathbf{d}) \times \mathbf{m}\rangle\langle\mathbf{x}, \mathbf{a}+\mathbf{b}\rangle+\right. \\
& +\langle\mathbf{x},\langle\mathbf{a}, \mathbf{b}\rangle((\mathbf{c}-\mathbf{d}) \times \mathbf{m})-\langle\mathbf{c} \times \mathbf{d}, \mathbf{m}\rangle(\mathbf{a}+\mathbf{b})\rangle+\langle\mathbf{c} \times \mathbf{d}, \mathbf{m}\rangle\langle\mathbf{a}, \mathbf{b}\rangle),
\end{aligned}
$$

where $\bar{\varepsilon}(\mathbf{x})=\bar{\varepsilon}(\overrightarrow{O X}):=\varepsilon(X)$. This proves that the equioptic is a union of arcs of two cubic algebraic curves. Since the equation for the compoptic is the same as (2.1), but with $-\bar{\varepsilon}$, the second statement of the theorem is also proven.

Beitr. Algebra Geom. 54 (2013), 659-667.

(c) Á. Kurusa 
The cubic algebraic curves

$$
\begin{aligned}
&|\mathbf{x}|^{2}\langle\mathbf{x},(\mathbf{a}-\mathbf{b}) \times \mathbf{m}\rangle+\langle\mathbf{a} \times \mathbf{b}, \mathbf{m}\rangle|\mathbf{x}|^{2}-\langle\mathbf{x},(\mathbf{a}-\mathbf{b}) \times \mathbf{m}\rangle\langle\mathbf{x}, \mathbf{c}+\mathbf{d}\rangle+ \\
&+\langle\mathbf{x},\langle\mathbf{c}, \mathbf{d}\rangle((\mathbf{a}-\mathbf{b}) \times \mathbf{m})-\langle\mathbf{a} \times \mathbf{b}, \mathbf{m}\rangle(\mathbf{c}+\mathbf{d})\rangle+\langle\mathbf{a} \times \mathbf{b}, \mathbf{m}\rangle\langle\mathbf{c}, \mathbf{d}\rangle \\
&= \pm\left(|\mathbf{x}|^{2}\langle\mathbf{x},(\mathbf{c}-\mathbf{d}) \times \mathbf{m}\rangle+\langle\mathbf{c} \times \mathbf{d}, \mathbf{m}\rangle|\mathbf{x}|^{2}-\langle\mathbf{x},(\mathbf{c}-\mathbf{d}) \times \mathbf{m}\rangle\langle\mathbf{x}, \mathbf{a}+\mathbf{b}\rangle+\right. \\
&+\langle\mathbf{x},\langle\mathbf{a}, \mathbf{b}\rangle((\mathbf{c}-\mathbf{d}) \times \mathbf{m})-\langle\mathbf{c} \times \mathbf{d}, \mathbf{m}\rangle(\mathbf{a}+\mathbf{b})\rangle+\langle\mathbf{c} \times \mathbf{d}, \mathbf{m}\rangle\langle\mathbf{a}, \mathbf{b}\rangle)
\end{aligned}
$$

are called Apollonian curves [11] and denoted by $\mathcal{A}_{ \pm}$according to the sign of the right side of (2.2). As $\mathbf{x}=\mathbf{0}$ is a solution of equations (2.2) if and only if $\langle\mathbf{a} \times \mathbf{b}, \mathbf{m}\rangle\langle\mathbf{c}, \mathbf{d}\rangle= \pm\langle\mathbf{c} \times \mathbf{d}, \mathbf{m}\rangle\langle\mathbf{a}, \mathbf{b}\rangle$, we infer that both Apollonian curves pass through $A, B, C, D$ and $\left\{M_{0}\right\}:=A B \cap C D$, but each one passes either $\left\{M_{+}\right\}:=A C \cap B D$ or $\left\{M_{-}\right\}:=A D \cap B C$ with corresponding index ${ }^{(2: 2)}$. (See Figure 1.)
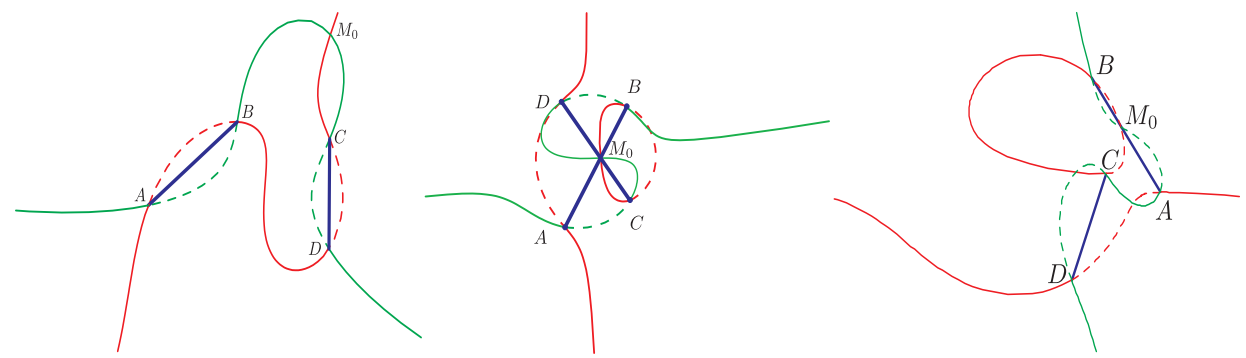

Figure 1. Apollonian curves $\mathcal{A}_{+}$and $\mathcal{A}_{-}$are shown in three configurations.

The equioptic and the compoptic are assembled from the arcs of these Apollonian curves and are drawn on Figure 1 by solid and dashed line, respectively.

Observations 2.2. The equioptic and compoptic subarcs of an Apollonian curve $\mathcal{A}$ follow each other alternately and changes happen, where $\mathcal{A}$ intersects one of the straight lines of the segments. Each Apollonian curve has a straight line asymptotic to it at its both "ends".

If an Apollonian curve intersects a straight line in more than three points, then, by Bézout's theorem ${ }^{(2: 3)}$, it contains that straight line as a component, and therefore it is reducible.

(2:2) Only the coincidence of the indices of $\mathcal{A}_{ \pm}$and $M_{ \pm}$is invariant, because changing the order of the endpoints of one of the segment changes both indices.

(2:3) Roughly speaking, Bézout's theorem [1] states that if two algebraic curves have more common points than the product of their degrees, then they have a common component.

Beitr. Algebra Geom. 54 (2013), 659-667.

(c) Á. Kurusa 
Theorem 2.3. If an Apollonian curve is reducible, then

(a) it consists of a nondegenerate circle and a straight line through its centre, or

(b) it consists of a degenerate circle and a straight line, or

(c) it consists of a nondegenerate equilateral hyperbola and the line at infinity, or

(d) it consists of two perpendicular straight lines and the line at infinity.

Theorem 2.3 is clearly stated in [11, p. 358, 1. -7] without proof, but there is a transparent proof of it in [12, Section 6] and a more detailed proof in [9, Section 2].

One needs to consider numerous cases to determine the configurations of the segments that yield reducible Apollonian curves. Therefore we only refer the reader to [10] for details.

Theorem 2.4. Let $\mathcal{A}$ be an Apollonian curve of the different nondegenerate segments $\overline{A B}$ and $\overline{C D}$.

(1) If $\mathcal{A}$ contains an open arc $\hat{\mathcal{H}}$ of a nondegenerate equilateral hyperbola $\mathcal{H}$, then the segments are opposite sides of a parallelogram and are separated by the branches of $\mathcal{H}$ if and only if they are the shorter edges in the parallelogram.

(2) If $\mathcal{A}$ contains an open arc $\hat{\mathcal{C}}$ of a circle $\mathcal{C}$, then it also contains a straight line $\ell$ passing through the centre of $\mathcal{C}$.

(3) If $\mathcal{A}$ contains an open segment $\hat{\ell}$ of a straight line $\ell$, then the segments are

(3.1) the reflections of each other with respect to $\ell$, or

(3.2) opposite sides of a kite ${ }^{(2: 4)}$ symmetric with respect to $\ell$, or

(3.3) adjoining sides of a kite symmetric with respect to $\ell$, or

(3.4) placed on $\ell$.

\section{Distinguishability of convex polygons}

We call an arc Apollonian if there is an Apollonian curve covering it. It is called irreducible if the covering Apollonian curve is irreducible.

Theorem 3.1. Let $\partial \mathcal{B}$ be the boundary of the convex open body $\mathcal{B}$ such that every arc of $\partial \mathcal{B}$ is either non-Apollonian or a segment or a hyperbolic arc or a circular arc of a disc containing $\mathcal{B}$ in its interior. Then any two convex open polygons in the interior of $\mathcal{B}$ can be distinguished by their visual angle functions given on $\partial \mathcal{B}$.

(2:4) A kite (a deltoid) is a quadrilateral that is symmetric with respect to at least one of the straight lines of its diagonals.

Beitr. Algebra Geom. 54 (2013), 659-667.

(c) Á. Kurusa 
Proof. If $X$ is an exterior point of the open convex polygon $\mathcal{P}$, then there are exactly two tangents of $\mathcal{P}$ trough $X$. We say that a diagonal (or an edge) of $\mathcal{P}$ can be seen at $X$ if the diagonal (or the edge, respectively) ends on both tangents. Observe that every point exterior to $\mathcal{P}$ sees at least a diagonal or an edge of $\mathcal{P}$.

Assume that $\mathcal{P}_{1}$ and $\mathcal{P}_{2}$ are open polygons inside $\mathcal{B}$ so that they subtend equal angles at every point of $\partial \mathcal{B}$.

Diagonals or edges of $\mathcal{P}_{1}$ and $\mathcal{P}_{2}$ that can be simultaneously seen at the points of a non-Apollonian arc in $\partial \mathcal{B}$ coincide, because the equioptic of two segments is Apollonian.

Diagonals or edges of $\mathcal{P}_{1}$ and $\mathcal{P}_{2}$ that can be simultaneously seen at the points of a segment $\mathcal{I}$ in $\partial \mathcal{B}$ coincide ${ }^{(3: 5)}$, because by statement (3) of Theorem 2.4 either they are on different sides of the straight line $\ell$ of segment $\mathcal{I}$ or they both intersect $\ell$, contradicting the convexity of $\mathcal{B}$.

Diagonals or edges $\overline{A B}$ and $\overline{C D}$ of $\mathcal{P}_{1}$ and $\mathcal{P}_{2}$ that can be simultaneously seen at the points of a hyperbolic $\operatorname{arc} \mathcal{I}$ in $\partial \mathcal{B}$ are, by statement (1) of Theorem 2.4, opposite sides of a parallelogram $\mathcal{Q}$ and either they are separated by the branches of the hyperbola $\mathcal{H}$ of $\operatorname{arc} \mathcal{I}$ or they both end on different branches of $\mathcal{H}$. In the first case, any tangent straight line $\ell$ of $\mathcal{I}$ separates $\overline{A B}$ and $\overline{C D}$, which contradicts convexity of $\mathcal{B}$. In the second case any tangent straight line $\ell$ of $\mathcal{I}$ intersects $\overline{A B}$ and $\overline{C D}$, which again contradicts the convexity of $\mathcal{B}$.

Let $\mathcal{I}$ be an arc in $\partial \mathcal{B}$ that is an arc of the bounding circle $\mathcal{C}$ of the disc $\mathcal{D}$ that contains $\mathcal{B}$ in its interior. Then the diagonals or edges $\overline{A B}$ and $\overline{C D}$ of $\mathcal{P}_{1}$ and $\mathcal{P}_{2}$ that can be simultaneously seen at the points of $\mathcal{I}$ coincide ${ }^{(3: 6)}$, because, by case (a) in the proof of statement (3) of Theorem 2.4, some of the points $A, B, C, D$ are necessarily outside of $\mathcal{D}$, contradicting $\mathcal{B} \subseteq \mathcal{D}$.

Summarizing, we have seen that the diagonals or edges of $\mathcal{P}_{1}$ and $\mathcal{P}_{2}$ that can be simultaneously seen at the points of $\partial \mathcal{B}$ coincide. Although there may be diagonals and edges of $\mathcal{P}_{i}(i=1,2)$ that can not be seen at any point of $\partial \mathcal{B}$. Any vertex of $\mathcal{P}_{i}(i=1,2)$ is the end of at least one such diagonal or edge, therefore the proof is complete.

Theorem 3.1 improves [4, Theorem 2.3 and Theorem 3.1] by allowing many more types of arcs. Nevertheless, if hyperbolic arcs were not allowed in Theorem 3.1, then the convexity condition could be dropped too.

Our next result drops convexity and analyticity from [4, Theorem 3.1].

(3:5) This is also proved with a different method in [4, Lemma 3.3.]

(3:6) This is also proved with a different method in [4, Lemma 2.2.]

Beitr. Algebra Geom. 54 (2013), 659-667.

(c) Á. Kurusa 
Theorem 3.2. If the boundary $\partial \mathcal{D}$ of the open domain $\mathcal{D}$ is differentiable by order seven, then any two convex closed polygons in the interior of $\mathcal{D}$ can be distinguished by their visual angle functions on $\partial \mathcal{D}$.

Proof. Let $\mathcal{P}_{1}$ and $\mathcal{P}_{2}$ be the two closed convex polygons in the interior of $\mathcal{D}$.

Assume that no diagonal or edge of $\mathcal{P}_{1}$ coincides with any diagonal or edge of $\mathcal{P}_{2}$ if they can be simultaneously seen at any point of $\partial \mathcal{D}$. Then every arc of $\partial \mathcal{D}$ is Apollonian, therefore every arc of $\partial \mathcal{D}$ is algebraic curve of degree at most 3 . As they join to each other by order 7 of differentiability (and they have two common cyclic points), Bézout's theorem implies that they all are covered by the same Apollonian curve $\mathcal{A}$, i.e., $\partial \mathcal{D} \subseteq \mathcal{A}$. This means that every simultaneously seen pair of edges and diagonals has equal or supplementary shadow picture at every point of $\partial \mathcal{D} \subseteq \mathcal{A}$.

A straight line of any edge or diagonal of $\mathcal{P}_{1}$ and $\mathcal{P}_{2}$ intersects $\partial \mathcal{D} \subseteq \mathcal{A}$ in at least two points. In such points the shadow pictures of the corresponding edge or diagonal of $\mathcal{P}_{1}$ and $\mathcal{P}_{2}$ vanish, hence the straight lines of the simultaneously seen pairs of edges and diagonals of $\mathcal{P}_{1}$ and $\mathcal{P}_{2}$ intersect $\partial \mathcal{D} \subseteq \mathcal{A}$ in at least two common points. Therefore any simultaneously seen pair of edges and diagonals is on a common straight line $\ell$, hence their Apollonian curve is reducible. Thus, by Theorem 2.3, $\partial \mathcal{D}$ is a circle symmetric to $\ell$. By Lemma 2.1. in [4], this implies that every simultaneously seen pair of edges and diagonals of $\mathcal{P}_{1}$ and $\mathcal{P}_{2}$ coincides. This contradicts our assumption that no diagonal or edge of $\mathcal{P}_{1}$ coincides with any diagonal or edge of $\mathcal{P}_{2}$.

Thus there is a diagonal or edge $\overline{A B}$ of $\mathcal{P}_{1}$ that is also a diagonal or edge of $\mathcal{P}_{2}$, which can be seen at the points of an $\operatorname{arc} \mathcal{I}$ in $\partial \mathcal{D}$.

Let $g$ be that arc-length parametrization of $\partial \mathcal{D}$ for which $\mathcal{I} \subseteq\{g(s): s \leq 0\}$ and $X:=g(0)$ is the end point of $\mathcal{I}$ in counterclockwise direction.

Denote the tangent line, the tangent vector and the curvature of the curve $\partial \mathcal{D}$ at $g(s)$, by $\ell(s), \mathbf{v}(s)$ and $\kappa(s)$, respectively. Let the tangents of $\mathcal{P}_{i}$ through $g(s)$ be denoted by $\ell_{i}^{+}(s)$ and $\ell_{i}^{-}(s)$, so that their respective unit directional vectors $\mathbf{v}_{i}^{+}(s)$ and $\mathbf{v}_{i}^{-}(s)$ are in the same side of $T(s)$ and $\mathbf{v}_{i}^{-}(s)$ is a positive linear combination of $\mathbf{v}_{i}^{+}(s)$ and $\mathbf{v}(s)$. Let $\alpha_{i}(s)$ and $\beta_{i}(s)$ be the angles of $\mathbf{v}_{i}^{+}(s)$ and $\mathbf{v}_{i}^{-}(s)$ to $\mathbf{v}(s)$, respectively. Then $0<\alpha_{i}(s)<\beta_{i}(s)<\pi$, and $\nu_{i}(s):=\beta_{i}(s)-\alpha_{i}(s)$ is the shadow picture of $\mathcal{P}_{i}$ at the source $g(s)$ obviously.

Let $\mathcal{A}_{i}(s)=\mathcal{P}_{i} \cap \ell_{i}^{+}(s)$ and $\mathcal{B}_{i}(s)=\mathcal{P}_{i} \cap \ell_{i}^{-}(s)$. Define the points $A_{i}^{ \pm}(s):=$ $\lim _{x \searrow 0} \mathcal{A}_{i}(s \pm x)$ and $B_{i}^{ \pm}(s):=\lim _{x \searrow 0} \mathcal{B}_{i}(s \pm x)$. Finally let $a_{i}^{ \pm}(s)=\left|A_{i}^{ \pm}(s)-g(s)\right|$ and $b_{i}^{ \pm}(s)=\left|B_{i}^{ \pm}(s)-g(s)\right|$. If $A_{i}^{+}(s)=A_{i}^{-}(s)$, then let $A_{i}(s)=A_{i}^{+}(s)$ and $a_{i}(s)=a_{i}^{+}(s)$, and, similarly, if $B_{i}^{+}(s)=B_{i}^{-}(s)$, then let $B_{i}(s)=B_{i}^{+}(s)$ and $b_{i}(s)=b_{i}^{+}(s)$. 
Let $a_{i}:=a_{i}^{+}(0)-a_{i}^{-}(0)$ and $b_{i}:=b_{i}^{+}(0)-b_{i}^{-}(0)$, and note that one of these should be positive.

Assume that the edge or diagonal $\overline{A_{1} B_{1}}$ of $\mathcal{P}_{1}$ and the edge or diagonal $\overline{A_{2} B_{2}}$ of $\mathcal{P}_{2}$ that can be seen simultaneously at the points $g(s)$ of $\partial \mathcal{D}$ for small $s>0$ are different.

Since $\nu_{1} \equiv \nu_{2}$ on $\partial \mathcal{D}, \alpha:=\alpha_{1}(0)=\alpha_{2}(0)$ and $\beta:=\beta_{1}(0)=\beta_{2}(0)$, we have

$$
\frac{\sin \beta}{b+b_{1}}-\frac{\sin \alpha}{a+a_{1}}=\dot{\nu}_{1}(0+)=\dot{\nu}_{2}(0+)=\frac{\sin \beta}{b+b_{2}}-\frac{\sin \alpha}{a+a_{2}}
$$

and

$$
\begin{aligned}
\frac{\sin 2 \beta}{\left(b+b_{1}\right)^{2}} & -\frac{\sin 2 \alpha}{\left(a+a_{1}\right)^{2}}-\kappa\left(\frac{\cos \beta}{b+b_{1}}-\frac{\cos \alpha}{a+a_{1}}\right) \\
& =\ddot{\nu}_{1}(0+)=\ddot{\nu}_{2}(0+)=\frac{\sin 2 \beta}{\left(b+b_{2}\right)^{2}}-\frac{\sin 2 \alpha}{\left(a+a_{2}\right)^{2}}-\kappa\left(\frac{\cos \beta}{b+b_{2}}-\frac{\cos \alpha}{a+a_{2}}\right),
\end{aligned}
$$

by $[6$, Lemma 1$]$.

Introducing $\bar{a}=a+a_{1}, \bar{b}=b+b_{1}, x=a_{2}-a_{1}$ and $y=b_{2}-b_{1}$, we obtain

$$
\frac{\sin \beta}{\bar{b}}-\frac{\sin \alpha}{\bar{a}}=\frac{\sin \beta}{\bar{b}+y}-\frac{\sin \alpha}{\bar{a}+x},
$$

$$
\frac{\sin 2 \beta}{\bar{b}^{2}}-\frac{\sin 2 \alpha}{\bar{a}^{2}}-\kappa\left(\frac{\cos \beta}{\bar{b}}-\frac{\cos \alpha}{\bar{a}}\right)=\frac{\sin 2 \beta}{(\bar{b}+y)^{2}}-\frac{\sin 2 \alpha}{(\bar{a}+x)^{2}}-\kappa\left(\frac{\cos \beta}{\bar{b}+y}-\frac{\cos \alpha}{\bar{a}+x}\right) .
$$

Expressing $v=1 /(\bar{b}+y)$ by $u=1 /(\bar{a}+x)$ from the first equation, and putting it into the second one, the second equation clearly becomes a second order equation for $u$, and therefore it has at most two solutions. One of these solutions is obviously $u=1 / \bar{a}$, which means $x=0$; this is excluded by our assumption.

Thus, $a_{1}$ and $b_{1}$ determine $a_{2}$ and $b_{2}$ uniquely, and the sign of $a_{2}-a_{1}$ agrees with the sign of $b_{2}-b_{1}$ by (3.1). Assume that $a_{2}<a_{1}$ and therefore $b_{2}<b_{1}$.

Let $\mathcal{J} \subset\{g(s): 0 \leq s\}$ be the maximal arc of $\partial \mathcal{D}$ at the points of which $\overline{A_{1} B_{1}}$ and $\overline{A_{2} B_{2}}$ can be simultaneously seen. Let $Y=g(y)$ be the end point of $\mathcal{J}$ different from $X$.

Let $\mathcal{A}_{+}$be the Apollonian curve of the segments $\overline{A_{1} B_{1}}$ and $\overline{A_{2} B_{2}}$ that goes through $X$. The straight lines $A_{1} B_{1}$ and $A_{2} B_{2}$ divide the plane into four quadrants. We denote the quadrant that contains $X=M_{+}$by $\mathcal{Q}_{-2}$, and the quadrant that has in its boundary the segments $\overline{A_{1} B_{1}}$ and $\overline{A_{2} B_{2}}$ by $\mathcal{Q}_{1}$. The remaining two quadrants are respectively denoted by $\mathcal{Q}_{2}$ and $\mathcal{Q}_{-1}$, according to the index of the quadrant not adjacent with them. Denoting the asymptotic of $\mathcal{A}_{+}$by $\ell$, we have the following observations: 
$\left(o_{1}\right) \ell$ goes through the midpoints of the segments $\overline{A_{1} A_{2}}$ and $\overline{B_{1} B_{2}}$; therefore the segments are on different sides of $\ell$;

$\left(o_{2}\right) \quad \ell$ is tangent to $\mathcal{A}_{+}$at the infinity; therefore it can intersect $\mathcal{A}_{+}$in at most one point (if $\ell$ intersected $\mathcal{A}_{+}$in two points, then it is a component of $\mathcal{A}_{+}$, and therefore the segments form a configuration given in statement (3) of Theorem 2.4, hence $\ell$ passes through exactly one-one endpoint of both segments that contradicts the condition $a_{2}<a_{1}$ );

$\left(o_{3}\right)$ As the arc $\mathcal{I}_{+}$of $\mathcal{A}_{+}$that goes through $M_{+}$is a part of the equioptic, it is covered by the compoptic in $\mathcal{Q}_{1}$;

$\left(o_{4}\right)$ The arc $\mathcal{I}_{0}$ of $\mathcal{A}_{+}$that goes through $M_{0}$ is a part of the equioptic, and in a neighbourhood of $M_{0}$ it is in $\mathcal{Q}_{1} \cup \mathcal{Q}_{-1}$.

We conclude that $\mathcal{I}_{+}$cannot pass $M_{0}$, whence it must go through $A_{2}$ and $B_{2}$. If $\mathcal{I}_{+}$left the quadrant $\mathcal{Q}_{1}$ it could only do that through $A_{1}$ or $B_{1}$, but then it has two intersections or one double intersection with $\ell$, contradicting $\left(o_{2}\right)$. Thus the arc $\mathcal{I}_{+}$is in $\mathcal{Q}_{-2} \cup \mathcal{Q}_{1}$, and therefore it cannot reach infinity, because the ideal points of $\ell$ are in $\mathcal{Q}_{-1} \cup \mathcal{Q}_{1}$ but $\mathcal{I}_{+}$is covered by the compoptic in $\mathcal{Q}_{1}$, while $\mathcal{A}_{+}$ is covered by the equioptic at infinity.

Thus, $\mathcal{I}_{+}$is the bounded component of $\mathcal{A}_{+}, \mathcal{I}_{0}$ is the unbounded component of $\mathcal{A}_{+}$, the points $A_{1}$ and $B_{1}$ are on $\mathcal{I}_{0}$, and the points $A_{2}$ and $B_{2}$ are on $\mathcal{I}_{+}$. Further, by [12, Proposition 7.1], the so-called middle line $\ell_{m}$ separates $\mathcal{I}_{+}$and $\mathcal{I}_{0}$, that is, the components $\mathcal{I}_{+}$and $\mathcal{I}_{0}$ are on different sides of $\ell_{m}$. Hence the configuration is much like the one seen on Figure 2.

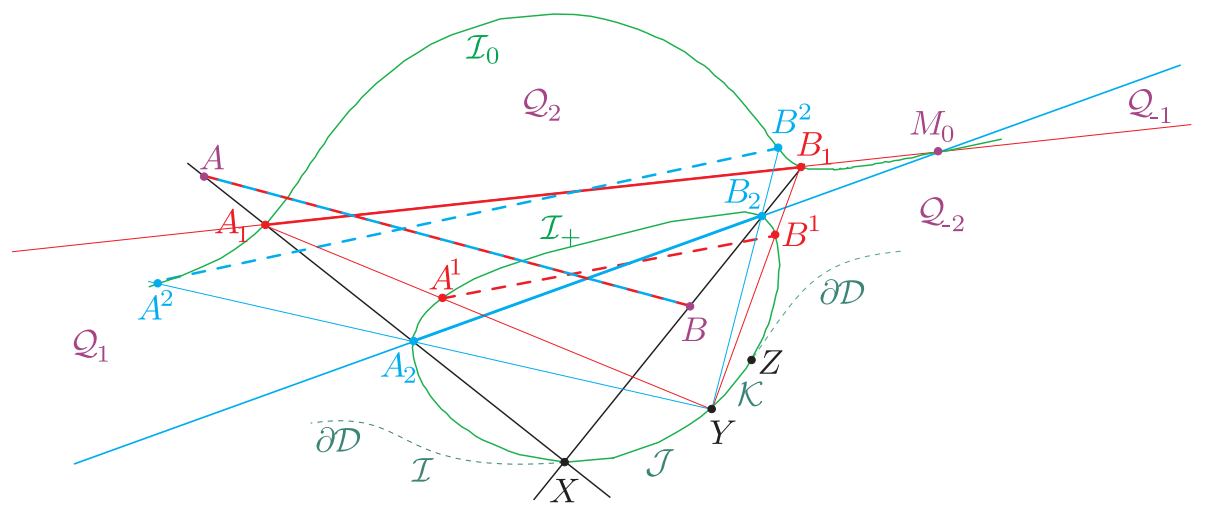

Figure 2. $\mathcal{I}_{+} \cup \mathcal{I}_{0}$ is the Apollonian curve $\mathcal{A}_{+}$.

Let $\overline{A^{i} B^{i}}$ be the edge or diagonal of the polygon $\mathcal{P}_{i}(i=1,2)$ that is seen at the points $g(s)$ for small enough $s>y$. Then

$A^{1} \neq A_{1}, A^{2} \neq A_{2}$ and $A^{1} \neq A^{2}, \quad$ and $\quad B^{1} \neq B_{1}, B^{2} \neq B_{2}$ and $B^{1} \neq B^{2}$,

Beitr. Algebra Geom. 54 (2013), 659-667.

(c) Á. Kurusa 
because $Y \neq X, A_{1} \neq A_{2}$ and $B_{1} \neq B_{2}$, and therefore $\overline{A^{1} B^{1}} \not \equiv \overline{A^{2} B^{2}}$. Thus the arc $\mathcal{K} \subseteq\{g(s): y \leq s\}$ of $\partial \mathcal{D}$ at the points from which the polygons $\mathcal{P}_{1}$ and $\mathcal{P}_{2}$ show up as $\overline{A^{1} B^{1}}$ and $\overline{A^{2} B^{2}}$ is Apollonian.

Since $\partial \mathcal{D}$ is of class $C^{7}$, both $\operatorname{arcs} \mathcal{J}$ and $\mathcal{K}$ are on the same Apollonian curve $\mathcal{A}_{+}$, hence $\left\{A^{i}\right\}=\left(\mathcal{A}_{+} \cap A_{i} Y\right) \backslash\left\{A_{i}, Y\right\}$ and $\left\{B^{i}\right\}=\left(\mathcal{A}_{+} \cap B_{i} Y\right) \backslash\left\{B_{i}, Y\right\}$ $(i=1,2)$. This implies that $A^{1}, B^{1} \in \mathcal{I}_{+}$and $A^{2}, B^{2} \in \mathcal{I}_{0}$ are on different sides of $\ell_{m}$, therefore at the points of $\mathcal{J}$ the polygons $\mathcal{P}_{1}$ and $\mathcal{P}_{2}$ show up with their diagonals $\overline{A_{1} B^{1}}$ and $\overline{A^{2} B_{2}}$, respectively.

This contradicts the consequence of our initial assumption that the different segments $\overline{A_{1} B_{1}}$ and $\overline{A_{2} B_{2}}$ can be simultaneously seen at the points of $\mathcal{J}$.

Thus, the segments $\overline{A_{1} B_{1}}$ and $\overline{A_{2} B_{2}}$ cannot be different, whence the statement of the theorem follows by induction.

\section{References}

[1] E. Brieskorn and H. Knörrer, Plane Algebraic Curves, Birkhäuser Verlag, Basel, 1986.

[2] R. J. Gardner, Geometric Tomography, Encyclopedia of Math. and its Appl. 58, Cambridge University Press, Cambridge, 2006 (first edition in 1996).

[3] J. Kincses, The determination of a convex set from its angle function, Discrete Comput. Geom. 30 (2003), 287-297.

[4] J. Kincses and Á. Kurusa, Can you recognize the shape of a figure from its shadows?, Beitr. Algebra Geom. 36 (1995), 25-34.

[5] J. Kincses, An example of a stable, even order quadrangle which is determined by its angle function, Discrete Geometry, in honor of W. Kuperberg's 60th birthday (ed.: A. Bezdek), CRC Press (Marcel Dekker), New York - Basel, 2003, 367-372.

[6] Á. Kurusa, You can recognize the shape of a figure by its shadows!, Geom. Dedicata 59 (1996), 103-112.

[7] Á. Kurusa, The shadow picture problem for nonintersecting curves, Geom. Dedicata 59 (1996), 113-125.

[8] Á. Kurusa, Is a convex plane body determined by an isoptic?, Beitr. Algebra Geom. 53 (2012), 281-294.

[9] Á. Kurusa, Equioptics of segments: generalizing Apollonius' theorem, Polygon, to appear (in Hungarian: "Szakaszok ekvioptikusai: Apollóniosz tételének általánositása").

[10] Á. Kurusa, Visual distinguishability of segments, manuscript.

[11] P. Pamfilos and A. Thoma, Apollonian cubics: An application of group theory to a problem in Euclidean geometry, Mathematics Magazine 72 (1999), 356-366. 
[12] P. Pamfilos, Theory of Isoptic Cubics, Help file of Isoptikon program that is freely available at http://www.math.uoc.gr/ pamfilos/\#iso , 1998.

Á. Kurusa, Bolyai Institute, University of Szeged, Aradi vértanúk tere 1., H-6720 Szeged, Hungary; e-mail: kurusa@math.u-szeged.hu 